\title{
TABLE OF LEGISLATION
}

\section{INTERNATIONAL TREATIES}

1968 Brussels Convention on jurisdiction and the enforcement of judgments in civil and commercial matters (consolidated version, OJ L 299, 31.12.1972, p 32) $12.004,12.005$ 12.006

Art 6(1) 12.074

Art 21 12.103

Art 22 12.103

Convention on jurisdiction and the enforcement of judgments in civil and commercial matters done at Lugano on 16 September 1988 (OJ L 319, 25.11.1988, p 9) 12.004

Convention on jurisdiction and the recognition and enforcement of judgments in civil and commercial matters done at Lugano on 30 October 2007 (OJ L 339, 21.12.2007, p 3) 12.004

Art 69(4) 12.004

Art 69(5) 12.004

\section{COUNCIL OF EUROPE}

European Convention for the Protection of Human Rights and Fundamental Freedoms 1.12

Art 6 $1.12,5.132,11.184$

Art 13 11.2

Protocol 1, Art 1 11.254

\section{EUROPEAN UNION}

Treaty establishing the European Community

Art 3 5.17

Art 10 1.01

Art 61 13.03

Art 65 13.03

Treaty on European Union (consolidated version, OJ C 115, 9.5.2008, p 13) Art 4(3) $1.01,4.107,5.17$

Art 6(1), (3) 1.12

Treaty on the Functioning of the European Union (consolidated version, OJ C 115, 9.5.2008, p 13)

Art 34 $1.07,1.09,1.15$

Art 45(3) 1.14

Art 49 1.07

Art 56 $109,1.15$

Art 101 ......... 0.01, 0.02, 0.09, 0.14, 1.05, $1.06,1.07,1.09,1.10,1.15,1.18$, 1.20, 2.01, 2.02, 2.06, 2.07, 2.08, $2.14,2.34,2.37,2.39,2.50,2.51$ $2.83,3.12,3.51,3.75,4.104,5.10$ 5.63, 7.17, 7.19, 7.20, 7.24, 7.81, 8.13, 9.31, 9.54, 10.01, 10.14, 10.39, 11.90, 12.08, 12.09, 12.78, 13.18,

13.35

Art 101(1) ..... 0.02, 2.09, 2.12, 2.14, 2.64, $5.14,5.16,5.18,11.128$

Art 101(2) $2.08,2.54,2.56$ Art 101(3) .............. 0.09, 0.13, 2.12, 3.49 Art 102 ........ 0.01, 0.09, 0.14, 1.05, 1.06, $1.07,1.09,1.10,1.15,1.18,1.20$, 2.34, 2.37, 2.39, 2.50, 2.51, 2.52, 2.83, 3.12, 3.51, 5.10, 5.63, 7.17, 7.18, 7.24, 7.91, 8.13, 10.01, 10.14, $10.39,11.90,12.09,13.18,13.35$

Art 157 1.09

Art 263 $9.19,11.45$

Art 267 $10.07,11.37$

Art 339 $4.76,5.91,5.92,5.105$

Art 340(2) $3.16,3.17,6.10$ 
Charter of Fundamental Rights of the European Union (OJ C 303, 14.12.2007, p 1) 1.12

Art 17(1) 11.254

Art 47 1.12

Agreement of 19 October 2005 between the European Community and the Kingdom of Denmark on jurisdiction and the recognition and enforcement of judgments in civil and commercial matters (OJ L 79, 21.3.2013, p 4) 12.05

Commission Regulation (EC) No 622/2008 of 30 June 2008 amending Regulation (EC) No 773/2004, as regards the conduct of settlement procedures in cartel cases (OJ L 171, 1.7.2008, p. 3) $4.03,4.186$

Art 16a(2) 4.116

Commission Regulation (EC) No 773/2004 of 7 April 2004 relating to the conduct of proceedings by the Commission pursuant to Articles 81 and 82 of the EC Treaty (OJ L 123, 27.4.2004, p. 18) 4.62

Art 10a 4.132

Art 15 4.62

Art 15(1) 4.62

Art 16a 4.62

Art 16(1) $5.93,5.94$

Art 16a(2) 4.63

Council Decision 2005/370/EC of 17

February 2005 concluding on behalf of the Community the Aarhus

Convention on access to information, public participation in decision-making and access to justice in environmental matters of 25 June 1998 (OJ L 124, 17.5.2005, p. 1) $11.73,11.75$

Art 4 11.58

Art 9 11.58

Art 9(1) $11.58,11.61,11.72$

Art 9(2) $11.59,11.60,11.61,11.66$ $11.70,11.72,11.74$

Art 9(3) $11.61,11.67,11.71,11.72$,
Council Directive 76/207/EEC of 9 February 1976 on the implementation of the principle of equal treatment for men and women as regards access to employment, vocational training and promotion, and working conditions (OJ L 39, 14.2.1976, p. 40) 2.73

Council Directive 85/374/EEC of 25 July 1985 on the approximation of the laws, regulations and administrative provisions of the Member States concerning liability for defective products (OJ L 210, 7.8.1985, p 29) $2.75-2.82$

Recital 11 $2.82,2.85$

Council Directive 93/13/EEC of 5 April 1993 on unfair terms in consumer contracts (OJ L 95, 21.4.1993,p 29)

Art 7(1), (2) 11.49

Council Regulation 17/62/EEC (first regulation implementing Articles 85 and 86 of the Treaty) (OJ L 13, 21.2.1962, p 204) 0.09

Art 19(3) 5.16

Council Regulation (EEC) No 4064/89 of 21 December 1989 on the control of concentrations between undertakings

(OJ L 395, 30.12.1989, p 1) ....... 13.15

Council Regulation (EC) No 44/2001 of 22

December 2000 on jurisdiction and the recognition and enforcement of judgments in civil and commercial matters (OJ L 12, 16.1.2001, p 1) 12.04, 12.06, 12.124, $12.138-12.140$

Recital 19 12.04

Art 5(3) 2.60

Art 27 12.103

Art 28 $3.68,12.108$

Council Regulation (EC) No 1206/2001 of 28 May 2001 on cooperation between the courts of the Member States in the taking of evidence in civil or commercial matters (OJ L 174, 27.6.2001, p 1) ........... 4.35, 4.36, 4.37, $4.39,4.40,4.42,4.45,4.47$ 
Council Regulation (EC) No 1/2003 of 16 December 2002 on the implementation of the rules on competition laid down in Articles 81 and 82 of the Treaty (OJ L 1 , 4.1.2003, p 1) ............. 0.08, 0.09, 0.12, $4.186,5.89,8.17,10.07$

Recital 7 $0.11,5.128$

Recital 13 5.11

Art 2 $3.49,3.51$

Art 3 0.10

Art 3(1) 8.10

Art 3(2) $13.01,13.24$

Art 6 0.10

Art 7 $4.118,5.10$

Art 9 $4.117,5.09,5.10$

Art 15(1) $0.10,4.88,4.109,4.131$ 10.01, 10.02, 10.03, 10.06, 10.25, 10.27

Art 15(3) $10.04,10.06,10.13,10.14$ $10.21,10.27,10.34$

Art 16 $5.02-5.26,5.27$ Art 16(1) ....... 5.02-5.05, 5.24, 5.27, 5.65, $5.71,11.106$

Art 28(2) $5.92,5.105$

Art 30 $5.89,5.91,5.92,5.129$

Art 30(1) 5.88

Art 30(2) $5.88,5.90,5.94$

Directive 2000/35/EC of the European Parliament and of the Council of 29 June 2000 on combating late payment in commercial transactions (OJ L 200, 8.8.2000, p 35) 9.119, 9.120

Directive 2004/35/EC of the European Parliament and of the Council of 21 April 2004 on environmental liability with regard to the prevention and remedying of environmental damage (OJ L 143, 30.4.2004, p. 56)

Art 12 $11.62,1.64$

Art 12(1) 11.66

Art 13(1) 11.63

Art 13(2) 11.68

Directive 2004/48/EC of the European Parliament and of the Council of 29 April 2004 on the enforcement of intellectual property rights (OJ L 157 , 30.4.2004, p 45) 5.124

Recital 18 11.57

Recital 21 5.125

Art 4(1) 11.56

Art 8 5.125

Directive 2009/22/EC of the European Parliament and of the Council of 23 April 2009 on injunctions for the protection of consumers' interests (OJ L 110, 1.5.2009, p 30)

Art 4(1) 11.50

Directive 2011/7/EU of the European Parliament and of the Council of 16 February 2011 on combating late payment in commercial transactions (recast) (OJ L 48, 23.2.2011, p 1) 9.118, 9.119

Recital 15 9.118

Arts 2, 7 9.118

Directive 2014/104/EU of 26 November 2014 on certain rules governing actions for damages under national law for infringements of the competition law provisions of the Member States and of the European Union (OJ L 349, 5.12.2014, p 1) ........... 0.17, 2.35, 3.14, 3.50, 3.62, 3.83, 3.86, 3.132, 3.140, 3.141, 3.143, 3.144, 3.151, 3.152, $3.155,3.156,4.32,4.51,4.52,4.54$, 4.55, 4.57, 4.109, 4.113, 4.159, 4.172, 4.173, 4.174, 4.175, 4.178, 4.179, 4.190, 5.37, 5.38, 5.39, 5.72, 5.74, 5.75, 5.114, 5.117, 6.15, 7.35, 7.43, 7.44, 7.49, 7.53, 7.76, 8.08, 8.09, 8.15, 8.56, 8.64, 8.68, 9.56, 9.57, 9.74, 9.78, $9.80,9.81,9.83,9.85,9.86,9.93,9.98$, $9.110,9.117,10.26,11.41,11.43$ $11.259,11.268$

Recital 10 $6.17,8.10$

Recital 11 6.17

Recital 12 7.06, 9.111, 9.112, 9.116, 9.129

Recital 13 7.07

Recital 14 4.56

Recital 15 4.56, 4.60, 4.107, 4.109, 10.02 
Recital 16

Recital 17

Recital 18

Recital 23

Recital 25

Recital 26

Recital 27

Recital 28

Recital 30

Recital 31

Recital 32

Recital 33

Recital 34

Recital 35

Recital 36

Recital 37

Recital 38

Recital 39

Recital 40

Recital 41

Recital 44

Recital 47

Art 1(1)

Art 1(2)

Art 2(2)

Art 2(3)

Art 2(4)

Art 2(12)

Art 2(14)

Art 2(16)

Art 2(18)

Art 3

Art 3(1)

Art 3(2)

Art 3(3)

Art 5

Art 5(1)

Art 5(2)

Art 5(3)

Art 5(5)

Art 5(8)

Art 6 .... 4.112, 4.119, 4.120, 4.126, 4.129,

4.130

Art 6(5) 4.87, 4.116, 4.117, 4.127,

$4.133-4.135,11.255$

Art 6(6) $4.119,4.127,4.149,5.117$
Art 6(7) 4.116, 10.08

Art 6(8) 4.126

Art 6(9) $4.119,4.126$

Art 6(11) $10.09,10.10,10.11,10.12$ $10.13,10.15,10.35$

Art 7 $4.112,4.119,4.120,4.121$

Art 7(1) $4.119,11.258$

Art 7(2) $4.119,11.258$

Art 7(3) ..... 4.119, 11.255, 11.257, 11.258 Art 8(2) 4.61

Art 9 $4.118,5.27-5.37,5.83$

Art 9(1) ......... 5.30, 5.32, 5.35, 5.70, 5.76

Art 9(2) ….............. 5.31, 5.32, 5.35, 5.70

Art 10 $9.23,9.51$

Art 10(1) $3.30,9.04$

Art 10(2) ....... 3.53, 9.08, 9.09, 9.10, 9.12, $9.14,9.58,9.59,9.95$

Art 10(3) ................ 9.06, 9.20, 9.38, 9.58

Art 10(4) ..... 4.181, 9.06, 9.11, 9.15, 9.16, $9.21,9.57,9.60,9.97$

Art 11 $8.82,8.93$

Art 11(1) $8.45,8.46,8.71,8.81$ Art 11(2) ........ 8.70, 8.71, 8.72, 8.73, 8.75 Art 11(3) $8.73,8.75$

Art 11(4) ....... 8.60, 8.62, 8.63, 8.64, 8.81, $8.83,9.21,9.22$ Art 11(5) ........ 8.50, 8.62, 8.63, 8.71, 8.76 Art 11(6) $8.63,8.64$ Art 12 ..... 3.106, 3.108, 3.126, 3.127, 7.11 Art 12(1) $3.14,3.62,3.106$

Art 12(2) 3.62

Art 12(3) $3.22,7.03$

Art 12(5) $3.63,3.70,7.54$

Art 13 $3.31,3.46,3.52,3.56,3.57$, $3.71,3.111,3.114$

Art 14 3.108

Art 14(1) $3.50,3.51,3.52$

Art 14(2) ....... 3.55, 3.56, 3.57, 3.58, 3.96, 3.107

Art 15(1) $3.67,3.69,3.71$

Art 15(2) 3.160

Art 17(1) $3.63,7.52,7.54$

Art 17(2) $3.58,7.31,7.36,7.37,7.38$

7.40

Art 17(3) ......... 10.16, 10.22, 10.26, 10.36

Art 18(1) 9.24

Art 20 5.37 
Art 20(2) 5.37

Art 21(1) 0.17

Art 22 3.96

Art 23 0.17

Regulation (EC) No 1049/2001 of the

European Parliament and of the Council of 30 May 2001 regarding public access to European Parliament, Council and Commission documents (OJ L 145, 31.5.2001, p 43) ........ 4.68, 4.69, 4.73, 4.78, 4.86, 4.92, 4.112, $4.129,4.130$

Art 4 $4.70,4.85,4.88$

Art 4(2) $4.75,4.78,4.87$

Art 4(3) $4.78,4.83$

Art 6(5) 4.87

Art 7 4.68

Regulation (EC) No 2006/2004 of the

European Parliament and of the Council of 27 October 2004 on cooperation between national authorities responsible for the enforcement of consumer protection laws (OJ L 364, 9.12.2004, p 1)

Art 3(b) $11.51,11.55$

Art 3(c) $11.52,11.53$

Art 3(k) 11.51

Arts 21-27 11.52

Regulation (EC) No 864/2007 of the European Parliament and of the Council of 11 July 2007 on the law applicable to non-contractual obligations (Rome II) (OJ L 199, 31.7.2007, p 40)

Recital 6 13.25

Recital 21 13.31

Art 4 13.06-13.07, 13.31

Art 4(1), (2), (3) 13.07

Art 5 13.06

Art 6 13.06

Art 6(3) $13.08-13.42,13.40$

Art 6(3)(a) ....... 13.30, 13.32, 13.39, 13.41 Art 6(3)(b) ....... 13.12-13.28, 13.29, 13.32 Art 6(4) 13.10

Art 7 13.06

Art 8 13.06

Art 9 13.06
Regulation (EU) No 1215/2012 of the European Parliament and of the Council of 12 December 2012 on jurisdiction and the recognition and enforcement of judgments in civil and commercial matters (OJ L 351, 20.12.2012, p 1) ..... 2.62, 12.03, 12.04 $12.05,12.06,12.12,12.14,12.40$, 12.102

Recital 44 $3.72,12.117$

Art 1(1) 12.06

Art 1(2) $12.06,12.17$

Art 4 .... 12.06, 12.14, 12.48, 12.49, 12.94, $12.1222,13.13$

Art 4(1) 12.15

Art 6(1) $12.11,12.122$

Art 7 $12.122,12.122$

Art 7(2) $2.60,12.46,12.51-12.73$, $13.13,13.29,13.39,13.42$

Art 7(5) 12.47

Art 8(1) ....... 12.10, 12.49, 12.74-12.101, $12.103,12.114,13.13,13.14,13.42$

Art 9 12.122

Art 25 12.18. 12.19, 12.23, 12.24, $12.29,12.30,12.32,12.42$

Art 25(1) 12.17

Art 29 12.10, 12.70, 12.103, 12.104-12.111, 12.112, 12.113, $12.119,12.120,12.121,12.122$, $12.123,12.124,12.125,12.126$, $12.128,12.136-12.137,12.140$

Art 29(1) $12.103,12.136$

Art 29(3) $12.103,12.137$

Art 30 $3.72,3.160,12.10,12.102$, 12.103, 12.111, 12.112-12.121, 12.122, 12.124, 12.125, 12.128, 12.131, 12.136-12.137

Art 30(1) $12.116,12.118$

Art 30(2) $12.114,12.137$

Art 30(3) 12.103, 12.115-12.117,

$12.130,13.14$

Art 31(1) ......................................... 12.103

Art 33 ...... 12.122, 12.126, 12.127, 12.128

Art 34 $12.122,12.128$

Art 62(1) 12.15

Art 63(1) $12.13,12.16$ 


\section{NATIONAL}

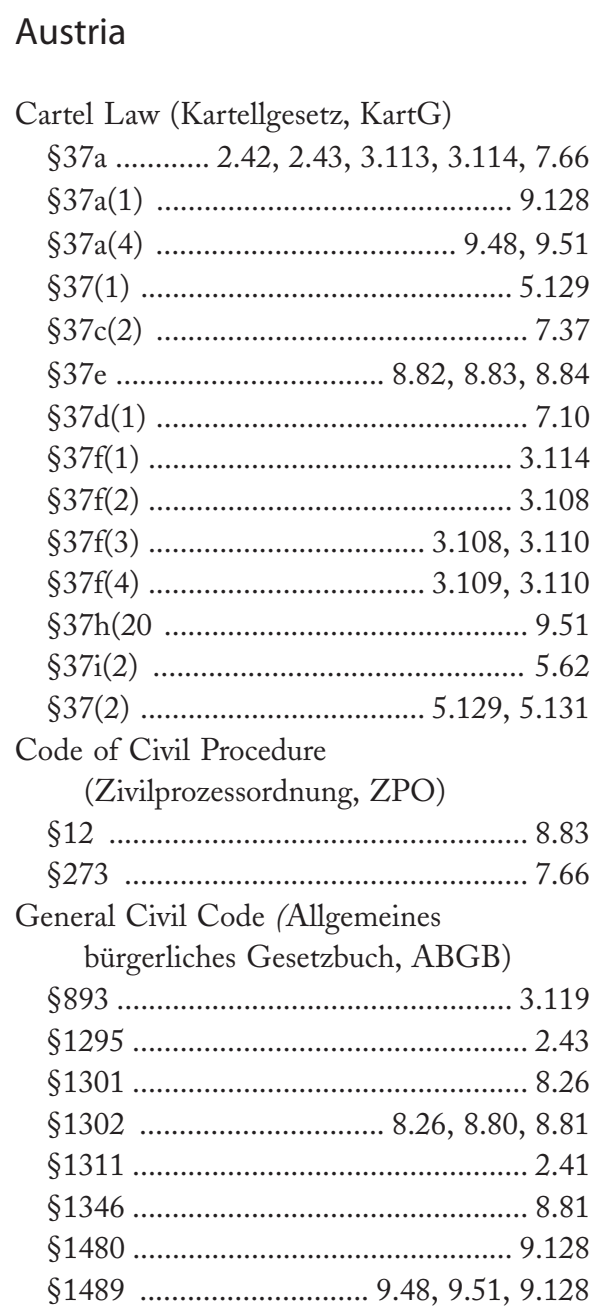

\section{England and Wales}

Civil Liability (Contribution) Act 1978

s $1(1)$ 8.48

s $2(1)$ 8.48

Civil Procedure Rules

Pt 19-II 4.15

Rule 19.6 11.119

Rule 19.6(1) $11.122,11.123$

Rule 19.6(4) $11.119,11.121$

Pt 19-III 11.119

Rule 19.10 11.124 Pt 31 11.125 4.13
Rule 31.17 4.15

Competition Act 1998 11.152

$$
\text { s 2(1) }
$$

s $18(1)$ $7.91,7.92$

s $47 \mathrm{~A}-\mathrm{E}$ 11.152 s 47A .... 2.32, 5.42, 5.43, 5.48, 5.51, 7.93, 9.25

s $47 \mathrm{~A}(8)$ $9.31,9.33$

s $47 \mathrm{~A}(9)$ $5.36,5.43$

s $47 \mathrm{~B}$ 2.32 s $47 \mathrm{E}$ 11.152

s $49 \mathrm{~A}-\mathrm{E}$ 5.42

s 58 3.79

s 60 3.79

s 60(1), (2) 7.79

s 14(5) 9.36 2003 4.13

Rule 19(2)(k) 9.26

Rule 31 9.27

Rule 31(1)

Competition Appeal Tribunal Rules 2015 $9.29,9.36,11.152$

Rule 19(2)(p) 4.13

Rules 71-72 10.18

Rule 78 11.155

Rule 78(2) 11.156

Rule 119 $9.26,11.134,11.153$

Rule 119(3) 9.27

Consumer Rights Act 2015 ......... 5.41, 9.29, $10.19,11.152-11.153$ Sch 8 $2.32,9.26,11.152$

\section{Damages Act 2017}

Part 2 3.83-3.86

Part 6 4.149

Part 7 4.149 para 9 3.84 para 11 3.85

Enterprise Act 2002 s 16(1), (4) …………......................... 10.18 s 19 11.127

European Communities Act 1972

$$
\text { s } 2(1)
$$

Foreign Limitation Periods Act 1984

$$
\text { s } 1
$$

Judicature Acts of 1873 and 1875 
Limitation Act 1980

s 2

s 32

9.34

\section{Finland}

Code of Judicial Procedure

(oikeudenkäymiskaari) 4.26

Competition Act (kilpailulaki,

948/2011) 2.68

s 20 ....

s 20(1) 2.68

s $49(2)$ 10.22

Competition Damages Act (laki

kilpailuoikeudellisista

vahingonkorvauksista,

1077/2016) ........ 2.68, 5.64, 7.42, 8.29, $8.86,8.87,9.62,10.22$

s $2(2)$

9.129

s $2(3)$

7.41

s 3

5.64

s $8(1)$

4.26

s 10 9.57

Interest Act (korkolaki, 633/1982)

s 4

s 7

9.130

s 12

9.129

9.130

Limitations Act (laki velan vanhentumisesta, $728 / 2003)$ 9.63

s $19(2)$ 8.86

Tort Liability Act (vahingonkorvauslaki, 412/1974) $8.85,9.63$

\section{France}

Civil Code (Code civile)

9.83

Art 1214

8.49

Art 1240 $1.16,2.44,2.45,2.64$

Art 1382 2.64

Art 2224 9.81

Art 2238

9.92

Art 2241 9.88

Civil procedure code (Code de procédure civile)

Art 9 4.19

Art 10

Art 11

4.23
Art 138 4.23

Art 145

4.20

Art 149

4.20

Art 275

4.22

Art 497 4.20

Code de commerce

4.173

Art L. 420-1 $2.46,2.64$

Art L. 420-2 2.46, 2.64

Art L. 420-3 2.46

Art L. 423-17 5.73

Art L. 462-3 $4.166,10.28,10.29$

Art L. 462-7 9.91

Art L. 463-6 $4.112,4.162$

Art L. 470-5 10.33

Art L. 481-2 $5.71,5.72,5.74,9.86$

Art L. 481-3 $7.10,7.44$

Art L. 483-2 4.23

Art L. 483-3 4.23

Art L. 483-5 4.1694 .172

Art L. 483-9 $4.170,4.178$

Consumer Code (Code de la consommation)

Art L. 622-1 ....... 11.189, 11.190, 11.192, $11.194,11.195$

Art L. 622-2 11.189

Art L. 623-1 11.194-11.200

Art L. 623-4 11.197

Art L. 623-24 11.198

Loi n ${ }^{\circ} 68-678$ relative à la communication de documents et renseignements d'ordre économique, commercial, industriel, financier ou technique à des personnes physiques ou morales étrangères, 26 July 1968 $4.38,4.39$, $4.42,4.44$

Loi n ${ }^{\circ} 2012-1270$ relative à la régulation économique outre-mer et portant diverses dispositions relatives aux outre-mer, 20 November 2012 .... 4.166

Ordonnance $n^{\circ}$ 2017-303 relative aux actions en dommages et intérêts du fait des pratiques anticoncurrentielles, 9 March 2017 $3.144,3.151$

Art 3 4.23, 4.170, 4.178, 5.71, 5.72, $5.74,7.10,7.44,9.86$

Art 3 4.166

Art 12 $4.168,9.87$ 


\section{Germany}

Act on Administrative Offenses

(Ordnungswidrigkeitengesetz,

OWiG) 2.34

$\S 46(1)$ and (3)

4.150

Civil Code (Bürgerliches Gesetzbuch, BGB) $\S 31$ 8.20

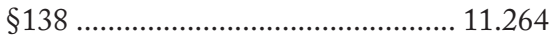

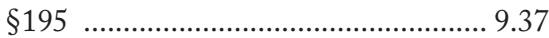

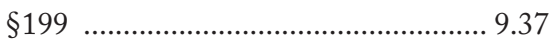

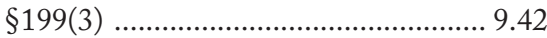

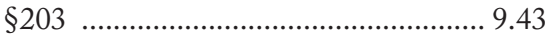

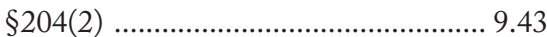

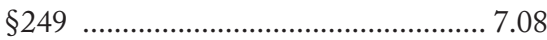

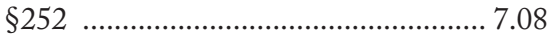

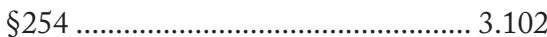

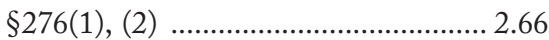

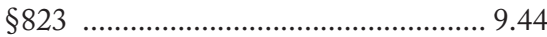

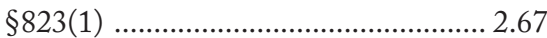

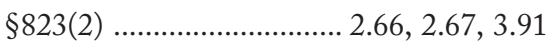

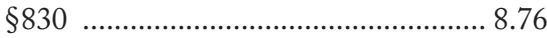

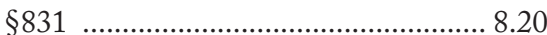

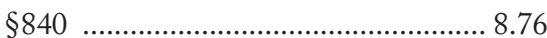

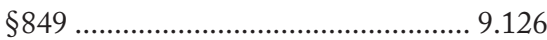

§852 ................................................... 9.47

Code of Civil Procedure

(Zivilprozessordnung, ZPO) .......... 2.36

$\S 68$

8.77

$\S 74$

8.77

$\S 101$

8.77

877

\$138(4) .................................. 8.78, 8.79

\$142 ….................................. 3.95, 4.25

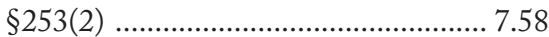

\$273(2) .............................................. 4.155

\$286(2) ............................................... 5.56

$\$ 287$.... 3.95, 7.36, 7.58, 7.59, 7.62, 7.63,

$7.65,7.66,11.263$

$\S 286$

6.34

Penal Procedural Code

(Strafprozessordnung, StPO) ........ 2.34 $\S 406$ 4.152

$\S 406 \mathrm{e}(1)$

4.150

$\S 406 \mathrm{e}(2)$

$4.151,4.152,4.153$

Penal Code (Strafgesetzbuch, StGB)

$\S \S 263,298$
Gesetz gegen Wettbewerbsbeschränkungen

(GWB) 2.34

$\S 1$......... 2.34, 2.37, 2.39, 2.40, 8.16, 8.17 $\S \S 19,20 \ldots \ldots \ldots \ldots \ldots \ldots \ldots . . . . . . . . . . . . . . . . .34,2.37,2.39$

$\$ 33$ $2.42,7.62$

$\S \S 33 \mathrm{a} \ldots \ldots \ldots . . . . .2 .34,2.36,3.87,8.19,9.47$

$\S 33 \mathrm{a}(1) \ldots \ldots \ldots . .2 .36,2.38,2.40,2.66,2.68$,

$5.58,8.16$

$\S 33 \mathrm{a}(3)$

$7.36,7.59$

$\S 33 \mathrm{a}(4)$

9.126

$\S 33 \mathrm{~b}$

$2.34,2,362.70,5.56,5.61$

$\$ 33 \mathrm{c}$

$2.34,2.36$

$\S 33 \mathrm{c}(2)$

3.96

$\S 33 \mathrm{e}$

8.76

$\S 33 \mathrm{~g}$ 4.24

$\S 33 \mathrm{~h}$ 9.38

$\S 33 \mathrm{~h}(6)$ $9.43,9.46$

§33(3)

$3.87,3.88,5.58,9.44,9.45$

$\S 33(4)$ $5.58,5.59,5.61,8.19,11.202$

$3 \S 33(5)$

$9.44,9.45$

$\S 34$

$7.10,11.202$

$\S 34 \mathrm{a}$ 11.202

$\S 81(3 a)$

$8.18,8.19$

$\S 89$ 2.36

$\S 89 \mathrm{~b}$ 4.24

$\S 89 \mathrm{c}$ 4.24

$\S 89 \mathrm{c}(5)$ 4.152

$\S 90(2),(5)$ 10.20

7. GWB-Novelle ....................................... 2.37

9. GWB-Novelle ...... 2.35, 4.24, 4.156, 9.38

\section{Hungary}

Competition Act 7.49

Italy

Constitution

Art 10(2)

Civil Code (Codice civile)

Art 1223 7.13

Art 1226 $7.13,7.68$

Art 1227

Art 1968

Art 2055

Art 2947 9.94 
Civil Procedure Code

Arts 39, 40 3.160

Art 96 $10.35,10.38$

Arts 210, 213 4.32

Consumer Code (Codice del consume)

Art 2(2) 3.162

Art 140(9) 3.162

Art 140-bis 11.214, 11.215, 11.216

Decreto legislativo no 3, 19 January 2017 ...... 3.157, 4.180, 5.77, 5.80, 7.46 $7.47,8.95,10.37,11.216$, Art 1(1)

11.216

Art 1(2) 7.11

Art 4(7) 10.35

Art 4(8) 4.181

Art 7 $5.77,5.79$

Art 7(1) $5.76,5.78,5.81$

Art 7(2) 5.81

Art 8 9.94

Art 8(1) 9.95

Art 8(2) 9.97

Art 9 $8.93,8.96$

Art 10 3.157

Art 10(1) 3.157

Art 10(2) 3.158

Art 10(3)

Art 11 $3.157,3.160$

Art 12 $3.157,3.160$

Art 13 3.157

Art 14(1) 7.13

Art 14(2) $5.78,7.45$

Art 14(3) 10.36

Art 18 11.217

L. $287 / 90$

Art 2 3.154

\section{Latvia}

Commercial Law (Komerclikums) Art 20 8.43

Competition Law (Konkurences likums) Art 21 $7.70,7.71$

\section{Lithunia}

Civil Code (Civilinis kodeksas) 9.78
Code of Civil Procedure (Lietuvos Respublikos civilinio proceso kodeksa) ... 4.27, 4.28, 5.69, 5.70, 10.24 Law on Competition (Lietuvos Respublikos konkurencijos i ştatymas (No VIII-1099, 23 March 1999)) ..... 4.159, 9.133

\section{Portugal}

Constitution

Art 52(3) 11.223

Civil Code (Código civil) (Decreto-Lei 47344/66, of 25 November 1966)

Art 306 9.102

Art 309 9.101

Art 498 $9.101,9.102$

Arts $562-564$ 3.168

Lei 83/95, of 31 August 1995 (Act on the right of procedural participation and actio popularis) 11.223

\section{Spain}

Civil Code (Código civil)

Art 1303 2.49

Art 1902 $2.47,2.48,2.50,9.98$

Law 15/2007, Ley de Defensa de la

Competencia, 3 July 2007 2.47

Law 3/2013, Ley de creación de la Comisión Nacional de los Mercados y la Competencia, 4 June 2013 ....... 2.47, $3.163,7.48$

Art 1 $2.50,2.51$

Art 2 $2.50,2.51$

Art 5(2) 10.39

Art 71 2.51

Art 72 $2.51,3.163,7.11$

Art 74 9.98

Art 75 5.84

Art 75(1) 5.83

Art 75(2) 5.84

Art 76(3) 7.48

Art 76(4) 10.49

Art 78(1) $2.51,7.11$

Art 78(3) 3.166, 3.167

Art 79(1) 3.163 
Art 79(2) 3.164

Law 1/2000, Ley de Enjuiciamiento Civil, 7 January 2000 2.47, 2.50, 3.163, $11.218,11.222$

Art 217 3.167

Real Decreto-ley 9/2017, 26 May 2017 $2.47,2.50,3.163$

\section{Sweden}

Act on class actions (Lag om

grupprättegång, 2002:599) 11.205 $11.207,11.208,11.212,11.213$

Competition Act (Konkurrenslag, 1993:20) $9.70,9.71,11.211$ $\$ 33$ 9.69

Competition Act (Konkurrenslag, 2008:579) $9.72,11.211$

Competition Damages Act

(Konkurrensskadelag,

2016:964) .... 9.74-9.76, 11.212, 11.213

Limitations Act (Preskriptionslag, 1981:130) $\S 2$ 9.64

$\S 5$ $9.67,9.68$

\section{The Netherlands}

Constitution (Grondwet)

Art 17 11.184

Act Establishing the ACM (Instellingswet Autoriteit en Markt)

Art 7 4.157

Act on Collective Settlement of Mass

Claims (Wet Collectieve Afhandeling

Massaschade) 11.174, 11.176, $11.178-11.186$

Civil Code (Burgerlijk Wetboek)

Art 2:26 11.175

Art 2:285 11.175

Art 3:305a 11.174-11.177
Art 3:310 9.53

Art 6:95-6:97 $3.135,3.136$

Art 6:100 3.130, 3.135, 3.136, 3.137

Art 6:1931 7.40

Code of Civil Procedure (Wetboek van Burgerlijke Rechtsvordering)

Art 44a(1) 10.21

Art 152 5.63

Art 161a 5.63

Art 843a 4.31

Implementation Act (Implementatiewet richtlijn privaatrechtelijke handhaving mededingingsrecht) 3.125, 3.132, 3.134, 11.268

\section{USA}

US Constitution

Art III 3.171

Cartwright Act 3.177

Class Action Fairness Act 2005 3.191 , 11.239-11.243

Clayton Act, 15 USC $\$ 16$

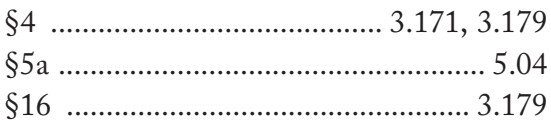

Federal Rules of Civil Procedure ....... 11.227

Rule 23 $3.178,11.228-11.238$

Rule 23(a) 11.228

Rule 23(b) 11.228, 11.230, 11.231, $11.232,11.233,11.237$

Rule 26(b) 4.184

Foreign Trade Antitrust Improvements Act of 1982 ......................................... 13.22

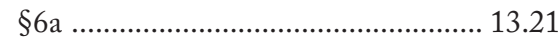

Sherman Act .......................................... 13.15

$\S 1$.................................................... 3.171

$\S 2 \ldots 3.171$

§7(1) .................................... 13.21, 13.22

§7(2) ................................................ 13.22 
David Ashton - 9781786430748 